\title{
Comment on: Virtual Reality, e-Learning, and Global Cardiac Surgical Capacity-Building
}

\author{
Omar Lattouf ${ }^{1}$ and Carlo Maria Rosati ${ }^{2}$ \\ ${ }^{1}$ Emory University School of Medicine \\ ${ }^{2}$ Indiana University School of Medicine
}

March 8, 2021

\begin{abstract}
Comment on: Virtual Reality, e-Learning, and Global Cardiac Surgical Capacity-Building

Title: Comment on: Virtual Reality, e-Learning, and Global Cardiac Surgical Capacity-Building

Running Title: Comment VR and e-Learning

Authors:
\end{abstract}

Carlo Maria Rosati, MD ${ }^{1}$; Omar M. Lattouf, MD PhD FACC FACS ${ }^{1}$

Affiliation:

${ }^{1}$ Mount Sinai Morningside, 1111 Amsterdam Avenue, New York, NY 10025

Corresponding Author:

Omar M. Lattouf, MD PhD FACC FACS

Professor of Cardiovascular Surgery, Mount Sinai Icahn School of Medicine

Professor Emeritus, Emory University

Mount Sinai Morningside, 1111 Amsterdam Avenue, New York, NY 10025

Email: omar.lattouf@mountsinai.org

No conflicts of interest.

Word count: 745

Disparities between higher and lower income countries in terms of accessibility to cardiac surgical care are appalling and reducing them is the commendable focus of interest of a growing series of initiatives.

Training a cardiac surgeon is notoriously a long and intensive process where "textbook knowledge", technical skills, clinical judgement and the ability to work within a team are all fundamental ingredients, that have to be mastered individually, as well as combined together.

The Authors of this manuscript ${ }^{1}$ are addressing the very important topic on how to train the next generation of cardiac surgeons from low- and middle-income countries. Besides more "traditional" challenges (such as the cost of travelling and living in high-income countries to receive on-site surgical training, and the high competition for cardiac surgery training positions), the ongoing COVID pandemic has added new ones, such as increased burdens on healthcare systems and international travel restrictions. 
The concepts offered by the Authors challenge the "age-old" training methodology, which has proven to be effective in producing safe, competent and innovative operators and educators. As cardiac surgeons have led their colleagues in all medical specialties in bringing new technologies within our system of training and practice, it is hard to convince ourselves to change. That said, the concepts put forth by the Authors must not be ignored, and the currently available technologies, together with the unexpected implications of the pandemic, can form the basis for the evolution of global cardiac surgical training.

Online learning and mentoring are powerful tools that are growing exponentially and should be well-known and accessible to all cardiac surgery trainees around the world. A few examples are:

- CTSNet

- Podcasts, such as the series by the Thoracic Surgery Residents Association and the Society of Thoracic Surgeons

- The new eBook by the Society of Thoracic Surgeons

- Smart phone apps with clinical practice guidelines, such as the American College of Cardiology/American Heart Association and the European Society of Cardiology

- Online simulation training modules/courses, such as "My Virtual Anastomosis" by Prof. Paul Sergeant

- Congenital Heart Academy

All major cardiac surgery meetings - including those of the Society of Thoracic Surgeons (STS), the American Association for Thoracic Surgery (AATS), the European Association for Cardio-Thoracic Surgery (EACTS), International Coronary Congress, etc. - are taking place in a virtual format. While such online format cannot replace the camaraderie and networking impact of in-person meetings, it is a great opportunity to reduce the cost to attend and to make such meetings accessible to every cardiac surgeon in every country of the world, without the need for long-distance travelling.

Interviews for training positions and jobs and meetings for shared clinical care and research collaborations are routinely done in a virtual format.

These are all new possibilities that can only help bring high-quality cardiac surgical training to low- and middle-income countries.

As far as actual "hands-on" training is concerned, the restrictions imposed by the COVID pandemic on international mobility are an added obstacle with an extremely high impact. While the development and distribution of vaccines holds promise for a "return to normalcy", it is not possible to foretell exactly for how long more such restrictions will be in place. We believe that, in the current situation, but also in the long term, it will be key to strengthen the role that experienced cardiac surgeons, who are already practicing in each country, have in training the next generations. It is essential to develop strong collaborations among national and international (such as STS, AATS and EACTS) cardiac surgical societies to "train the trainers" and give them the tools and support that are necessary to guarantee high-quality on-site hands-on training in each country. This is not an easy goal to accomplish. We congratulate the Authors on their commitment towards the fundamental topic of global cardiac surgery and we support and encourage such initiatives without any hesitation.

\section{Reference}

Vervoort D, Fiedler A. Virtual Reality, e-Learning, and Global Cardiac Surgical Capacity-Building. J Card Surg 2021. In Press. 